\title{
Perfil epidemiológico da mortalidade materna no estado do Espírito Santo durante o período de 2014 a 2018
}

\author{
Epidemiological profile of maternal mortality in the state of Espírito Santo from 2014 to \\ 2018
}

\section{Perfil epidemiológico de la mortalidad materna en el estado de Espírito Santo durante el período 2014 a 2018}

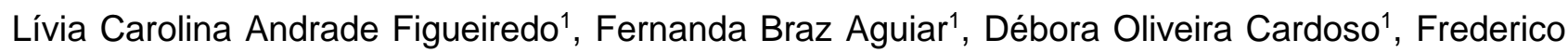
Bregunci de Castro ${ }^{1}$, Luiz Fernando de Almeida ${ }^{1}$, Anna Karolyna Rodrigues Cunha ${ }^{1}$, Bárbara Marques de Castro Lara², Gilberto de Souza², Larissa Mita ${ }^{1}$, Saulo Nascimento de Melo ${ }^{1 *}$.

\section{RESUMO}

Objetivo: Analisar a mortalidade materna no estado do Espírito Santo, no período de 2014 a 2018. Métodos: Este é um estudo epidemiológico do tipo ecológico transversal retrospectivo, foi utilizado o Sistema de Informação de Mortalidade para a coleta dos dados nas macrorregiões do estado, pelas variáveis: macrorregião de saúde, ano de ocorrência, faixa etária, cor e raça, escolaridade e estado civil. Resultados: As maiores taxas de mortalidade materna ocorreram em mulheres de pele parda (47.1\%), com 8 a 11 anos de escolaridade (39.0\%), faixa etária de 30 a 39 anos (44.2\%) e solteiras (49.0\%). A macrorregião Sul apresentou os maiores números de mortes maternas, sendo 386.1 óbitos por 100.000 habitantes. Conclusão: A mortalidade materna é um indicador da saúde feminina no mundo, por isso é fundamental o reforço da atenção à saúde de gestantes que se encaixam nos grupos expostos, assim como a implementação de políticas públicas direcionadas às gestantes.

Palavras-chave: Mortalidade materna, Epidemiologia, Saúde da mulher.

\begin{abstract}
Objective: The aim of this study was to analyze the maternal mortality in the state of Espírito Santo, from 2014 to 2018. Methods: This is a retrospective cross-sectional ecological epidemiological study, in which it was used the Mortality Information System to collect data in the state's macro-regions, variables: macro-region of health, year of occurrence, age group, color and race, education and marital status. Results: The highest rates of maternal mortality occurred in women with brown skin (47.1\%), with 8 to 11 years of schooling (39.0\%), aged between 30 and 39 years (44.2\%) and single (49.0\%). The Southern macro-region had the highest numbers of maternal deaths, with 386.1 deaths per 100,000 inhabitants. Conclusion: Maternal mortality is an indicator of women's health in the world, therefore it is essential to reinforce the health care of pregnant women who fit the exposed groups, as well as the implementation of public policies aimed at pregnant women.
\end{abstract}

Keywords: Maternal mortality, Epidemiology, Women's health.

\section{RESUMEN}

Objetivo: Analizar la mortalidad materna en el estado de Espírito Santo, de 2014 a 2018. Métodos: Se trata de un estudio epidemiológico transversal retrospectivo, utilizando el Sistema de Información de Mortalidad para recolectar datos en las macrorregiones del estado, por las variables: macrorregión de salud, año de ocurrencia, grupo de edad, color y raza, educación y estado civil. Resultados: Las mayores tasas de mortalidad materna se presentaron en mujeres de piel morena $(47,1 \%)$, con 8 a 11 años de escolaridad $(39,0 \%)$, de 30 a 39 años (44,2\%) y solteras (49,0\%). La macrorregión Sur tuvo el mayor número de muertes maternas, con 386,1 muertes por cada 100.000 habitantes. Conclusión: La mortalidad materna es un indicador de la salud de la mujer en el mundo, por eso es fundamental fortalecer la atención a la salud de las gestantes que encajan en los grupos expuestos, así como la implementación de políticas públicas dirigidas a las gestantes.

Palabras clave: Mortalidad materna, Epidemiología, La salud de la mujer.

${ }^{1}$ Universidade Federal de São João Del-Rei (USFJ), Divinópolis - MG. *E-mail: saulomelobio@hotmail.com

2 Universidade do Estado de Minas Gerais (UEMG), Divinópolis - MG. 


\section{INTRODUÇÃO}

Segundo a Organização Mundial da Saúde (OMS), o critério utilizado para se definir a mortalidade materna, é a morte da gestante durante ou até 42 dias após o término da gravidez, sendo esta relacionada ou agravada pela gestação, independente do tempo (WHO, 2012).

Os óbitos maternos que se relacionam às causas diretas são resultantes de complicações obstétricas na gravidez, no parto ou no puerpério em razão de tratamento impróprio, más técnicas ou omissões. As causas indiretas decorrem de doenças existentes antes da gravidez, ou que foram desenvolvidas durante a gravidez, sem uma relação com as causas diretas, mas devido ao agravamento dos efeitos fisiológicos da gravidez. Além disso, a definição para mortes maternas tardias são os óbitos por causas obstétricas diretas ou indiretas ocorridas no período entre pós-puerpério e até um ano após o término da gravidez (LAURENTI R, et al., 2008).

Dessa maneira, é necessário ressaltar que os óbitos maternos decorridos de complicações durante parto, puerpério e gravidez ocasionados por falha no acolhimento da gestante são potencialmente preveníveis. Sendo assim, as gestantes que apresentam risco em seu histórico de saúde e adquirem doenças ao longo da gestação devem ser acompanhadas com maior atenção desde o início da gestação (DIAS JMG, et al., 2014).

A mortalidade materna é um problema de saúde pública no Brasil e no mundo. Os índices de morte materna são indicadores da saúde da mulher, e de maneira indireta, também são indicadores da população geral (DIAS JMG, et al., 2014). Nesse sentido, a mortalidade materna se torna um parâmetro para que se possa avaliar a qualidade do serviço de saúde ofertado, para identificar situações de desigualdade e também contribui para avaliar os níveis de saúde e de desenvolvimento socioeconômico da população (MONTE AS, et al., 2018).

O esforço para a redução da mortalidade materna é um tema de aprofundamento social, que levou a uma compreensão sobre seus determinantes sociais, como: o papel da educação, a renda, o local de nascimento, os valores culturais e o grau de opressão ao qual a mulher está sujeita na sociedade (UNFPA, 2012). Assim, inserida em um contexto de saúde reprodutiva e de direitos sexuais, a mortalidade materna passou a ser entendida como um indicador de desenvolvimento social (SOUZA JP, 2015).

A partir desse entendimento, a redução da mortalidade materna foi incluída como uma das metas a serem atingidas dentro dos Objetivos de Desenvolvimento do Milênio (ODM). No ano de 2000 o objetivo foi a redução da taxa de mortalidade materna em 75\% até 2015 (UNDP, 2014). Posteriormente, para os anos de 2016 a 2030, o Objetivo de Desenvolvimento Sustentável (ODS), que substituiu o ODM, destacou novas metas, entre elas, a redução da razão de mortalidade materna global para menos de 70 mortes maternas por 100 mil nascidos vivos (SOUZA JP, 2015; SANTOS DR, et al., 2017). A ineficácia da assistência pré-natal e a falta de atenção ao parto e puerpério são frequentes no Brasil (NOGUEIRA LDP e OLIVEIRA GS, 2017). Um dos principais motivos que envolvem a baixa realização do pré-natal no país, está relacionada aos aspectos de ordem social da gestante, sendo este um fator agravante da mortalidade materna (GOIS EC, et al., 2019).

No Brasil, conhecer as especificidades regionais é importante, pois nelas há diferentes contextos socioepidemiológicos de ocorrências da mortalidade materna; isso permite a fomentação de políticas em saúde que visem uma melhoria na gestão do serviço e na qualidade da assistência prestada às mulheres (SANTOS DR, et al., 2017). Os estudos identificados na literatura são prioritariamente realizados nas grandes cidades e nas capitais do país (BRASIL, 2006).

Por isso, é importante ampliar a investigação para outros municípios e avançar na qualidade das informações e no conhecimento das causas de morte, para que seja possível realizar ações locais mais efetivas no controle das causas prevalentes e garantir uma destinação mais adequada de recursos públicos (MATIAS JP, et al., 2009). No Espírito Santo, há uma dependência espacial dos municípios que compõem a Região Metropolitana da Grande Vitória, por ser uma região com melhores indicadores socioeconômicos e com concentração dos serviços de saúde, em especial materno-infantis (ALVES KCG e ZANDONADE E, 2004). 
Dessa maneira, programas como a Rede Cegonha, instituído pelo Ministério da Saúde por meio da Portaria 1.459 de 24 de junho de 2011, pertencente ao Sistema Único de Saúde (SUS) e são fundamentais para a redução dos índices de morte materna. Este programa tem como finalidade implementar uma rede de cuidados para assegurar às mulheres o direito ao planejamento reprodutivo e a atenção humanizada à gravidez, ao parto e ao puerpério (BRASIL, 2011).

Nesse sentido, é importante rastrear grupos de vulnerabilidade social por meio da avaliação indicadores socioeconômicos, a fim de propor soluções que melhorem a qualidade de vida de gestantes e puérperas, minimizando a morte materna. Dito isso, o presente estudo tem o objetivo de analisar e comparar a taxa de mortalidade materna no estado do Espírito Santo, por meio das seguintes variáveis: macrorregião de saúde, ano de ocorrência, faixa etária, cor e raça, escolaridade e estado civil, no período de 2014 a 2018.

\section{MÉTODOS}

Foi realizado um estudo epidemiológico do tipo ecológico e de caráter transversal retrospectivo, com a utilização do Sistema de Informação Geográfica (SIG) para georreferenciamento dos óbitos maternos nas macrorregiões do estado do Espírito Santo (ES), abrangendo o período de 2014 a 2018. A abordagem de ordem social da mulher gestante será apresentada junto aos resultados.

Segundo a base de dados do Departamento de Informática do Sistema Único de Saúde (DATASUS) obteve-se as informações em saúde do estudo. Os dados utilizados na pesquisa foram obtidos pelo Sistema de Informação de Mortalidade (SIM) para as macrorregiões do estado e para cada ano do período citado. $O$ SIM, tem cobertura estimada de $85 \%$ dos óbitos ocorridos em todo o país, segundo dados demográficos (DIAS JMG, et al., 2014). Nos estados do Sul e Sudeste, a abrangência de notificações desse sistema é de aproximadamente $100 \%$, sobretudo nas capitais (LAURENTI R, et al., 2004).

Os óbitos foram coletados com base no Capítulo XV da lista de Classificação Internacional de Doenças (CID-10) que corresponde à gravidez, parto e puerpério, no qual foram selecionados somente a seção de óbitos maternos. Os dados referentes aos nascidos vivos foram coletados no Sistema de Informação de Nascidos Vivos (SINASC) utilizando a ferramenta de tabulação (TABNET) das Estatísticas Vitais do DATASUS, e foram selecionados por ano e por macrorregiões do Espírito Santo entre os anos de 2014 a 2018. Para a extração dos dados deste estudo foram selecionadas as seguintes varíaveis, disponíveis no DATASUS: macrorregião de saúde, ano de ocorrência, faixa etária, cor e raça, escolaridade (tempo de permanência na escola) e estado civil. As análises foram baseadas em dados de domínio público, de acesso irrestrito e sem identificação dos indivíduos.

A taxa de mortalidade materna nas macrorregiões de saúde do Espírito Santo foi calculada sob a seguinte fórmula: número de óbitos maternos por causas diretas e indiretas, dividido pelo número de nascidos vivos para cada ano e depois multiplicado por cem mil habitantes. Dessa maneira, foram calculadas as taxas de mortalidade materna para cada ano do período estudado. Essas informações foram processadas e apresentadas sob a forma de tabelas com a utilização do software Microsoft Excel 2019.

O georreferenciamento ou geoprocessamento da taxa de mortalidade materna nas macrorregiões do Espírito Santo foi realizado por meio do Sistema de Informação Geográfica (SIG). Em seguida, foi realizada a geolocalização quantitativa dos óbitos maternos tendo como unidades espaciais as macrorregiões de saúde, que foram feitas por meio do software ArcGIS 10.1. Os resultados foram apresentados por meio de mapa categorizado por macrorregião, estratificado pela incidência de mortalidade materna por 100.000 habitantes.

Este estudo foi realizado com dados de domínio público, de acesso irrestrito, e por isso é dispensado pela Comissão Nacional de Ética em Pesquisa (CONEP) de análise do sistema Comitê de Ética em Pesquisa(CEP)/CONEP.

\section{RESULTADOS}

De acordo com os dados coletados observou-se que foram registrados 172 óbitos de mães no estado do Espírito Santo entre os anos de 2014 e 2018, sendo o ano de 2014 com o maior apontamento de óbitos (29.1\%). Dentre esses óbitos foram estratificadas as variáveis com os dados descritivos de ordem social da gestante (Tabela 1). 
A análise referente a cor e raça mostrou maior prevalência de morte nas gestantes pardas (47.1\%). Quanto à escolaridade, foi notificado que a maioria das gestantes frequentaram escola durante 8 a 11 anos (39.0\%). Além disso, a faixa etária em que se teve o maior número de óbitos foi entre 30 a 39 anos $(44.2 \%)$, seguido da faixa de 20 a 29 anos (36.1\%). Finalmente, o estado civil em que se ocorreu a maioria dos óbitos foi o de mães solteiras $(49.0 \%)$.

Tabela 1 - Dados descritivos da mulher gestante.

\begin{tabular}{|c|c|c|c|c|c|c|}
\hline \multirow[b]{2}{*}{ Variáveis } & \multicolumn{5}{|c|}{ Anos } & \multirow[b]{2}{*}{$\begin{array}{l}\text { Total } \\
(\%)^{\star}\end{array}$} \\
\hline & $\begin{array}{l}2014 \\
(\%)^{\star}\end{array}$ & $\begin{array}{l}2015 \\
(\%)^{\star}\end{array}$ & $\begin{array}{l}2016 \\
(\%)^{\star}\end{array}$ & $\begin{array}{l}2017 \\
(\%)^{\star}\end{array}$ & $\begin{array}{l}2018 \\
(\%)^{\star}\end{array}$ & \\
\hline \multicolumn{7}{|l|}{ Raça/Cor } \\
\hline Branca & $9(18 \%)$ & $10(27.8 \%)$ & $6(28.6 \%)$ & 8 (24.2\%) & $6(18.8 \%)$ & 39 (22.7\%) \\
\hline Preta & 25 (50\%) & 15 (41.7\%) & 8 (38.1\%) & 16 (48.5\%) & 17 (53.1\%) & 26 (15.1\%) \\
\hline Parda & $10(20 \%)$ & $2(5.5 \%)$ & $4(19 \%)$ & 5 (15.2\%) & 5 (15.6\%) & $81(47.1 \%)$ \\
\hline Ignorado & $6(12 \%)$ & $9(25 \%)$ & $3(14.3 \%)$ & $4(12.1 \%)$ & 4 (12.5\%) & $26(15.1 \%)$ \\
\hline \multicolumn{7}{|c|}{ Escolaridade } \\
\hline 0 a 7 anos & 14 (28\%) & 5 (13.9\%) & 3 (14.3\%) & 7 (21.2\%) & 5 (15.6\%) & 34 (19.7\%) \\
\hline 8 a 11 anos & $15(30 \%)$ & $16(44,4 \%)$ & $6(28.6 \%)$ & 17 (51.5\%) & $13(40.6 \%)$ & $67(39.0 \%)$ \\
\hline 12 ou mais & $5(10 \%)$ & 2 (5.5\%) & $1(4.7 \%)$ & $3(9.1 \%)$ & $2(6.3 \%)$ & $13(7.6 \%)$ \\
\hline Ignorado & 16 (32\%) & $13(36.2 \%)$ & $11(52.4 \%)$ & $6(18.2 \%)$ & $12(37.5 \%)$ & $58(33.7 \%)$ \\
\hline \multicolumn{7}{|l|}{ Faixa Etária } \\
\hline 10 a 19 & $4(8 \%)$ & $6(16.7 \%)$ & $1(4.7 \%)$ & $6(18.2 \%)$ & $3(9.4 \%)$ & $20(11.6 \%)$ \\
\hline 20 a 29 & 25 (50\%) & 14 (38.9\%) & $5(23.8 \%)$ & $10(30.3 \%)$ & $8(25 \%)$ & $62(36.1 \%)$ \\
\hline 30 a 39 & $19(38 \%)$ & $12(33.3 \%)$ & $12(57.2 \%)$ & $16(48.5 \%)$ & $17(53.1 \%)$ & $76(44.2 \%)$ \\
\hline 40 a 49 & $2(4 \%)$ & $4(11.1 \%)$ & 3 (14.3\%) & $1(3 \%)$ & $4(12.5 \%)$ & 14 (8.1\%) \\
\hline \multicolumn{7}{|l|}{ Estado Civil } \\
\hline Solteira & 28 (56\%) & $22(61.1 \%)$ & $6(28.6 \%)$ & 15 (45.5\%) & $13(40.6 \%)$ & $84(49.0 \%)$ \\
\hline Casada & $13(26 \%)$ & $5(13.9 \%)$ & $9(42.8 \%)$ & $11(33.3 \%)$ & 9 (28.1\%) & 47 (27.3\%) \\
\hline Outros & $3(6 \%)$ & $2(5.5 \%)$ & $3(14.3 \%)$ & $6(18.2 \%)$ & $3(9.4 \%)$ & 17 (9.8\%) \\
\hline Ignorado & $6(12 \%)$ & 7 (19.5\%) & 3 (14.3\%) & $1(3 \%)$ & 7 (21.9\%) & 24 (13.9\%) \\
\hline Total & $50(29.1 \%)$ & $36(21.0 \%)$ & $21(12.2 \%)$ & 33 (19.1\%) & 32 (18.6\%) & 172 (100\%) \\
\hline
\end{tabular}

Legenda: * Porcentagem calculada referente aos valores anuais.

Fonte: Figueiredo LCA, et al., 2020; Dados Extraídos do Sistema de Informação de Mortalidade (SIM).

As taxas de mortalidade materna, a cada cem mil habitantes, foram estratificadas pelas quatro macrorregiões do Espírito Santo (Tabela 2). A partir disso, nota-se que a macrorregião Sul tem apresentado as maiores taxas de mortalidade materna no período do estudo, com redução considerável apenas em 2016, ano em que o Norte liderou o número de óbitos. A macrorregião Central apresenta uma redução gradativa das taxas de mortes de mães durante o período analisado, já a macrorregião metropolitana se mantém com a menor taxa de mortalidade materna quando comparada às outras macrorregiões do Espírito Santo. 
Tabela 2 - Taxa de mortalidade materna por ano segundo as macrorregiões de saúde, por 100.000 habitantes no Espírito Santo.

\begin{tabular}{ccccccc}
\hline Macrorregiões & $\mathbf{2 0 1 4}$ & $\mathbf{2 0 1 5}$ & $\mathbf{2 0 1 6}$ & $\mathbf{2 0 1 7}$ & $\mathbf{2 0 1 8}$ & Total \\
\hline Sul & 108.7 & 77.4 & 23.7 & 133.6 & 44.9 & 388.3 \\
Norte & 98.6 & 48.6 & 103.2 & 97.6 & 32.2 & 380.2 \\
Metropolitana & 77.1 & 64.4 & 26.2 & 28.0 & 74.2 & 269.9 \\
Central & 101.7 & 55.0 & 58.0 & 69.7 & 22.0 & 306.4 \\
\hline Total & 386.1 & 245.4 & 211.1 & 328.9 & 173.3 &
\end{tabular}

Fonte: Figueiredo LCA, et al., 2020; Dados Extraídos do Sistema de Informação de Mortalidade (SIM).

Nesse sentido, expressa-se a incidência de óbitos maternos durante o período de estudo, no qual em 2014 houve a maior taxa com 386.1 óbitos/100.000 habitantes, seguido da segunda maior taxa em 2017 com 328.9 óbitos/100.000 habitantes, no estado do Espírito Santo (Gráfico 1). Apesar desse pico em 2017, houve uma tendência de declínio da incidência de óbitos maternos durante todo o período estudado.

Gráfico 1 - Taxa de mortalidade materna por ano segundo as macrorregiões de saúde no estado do Espírito Santo.

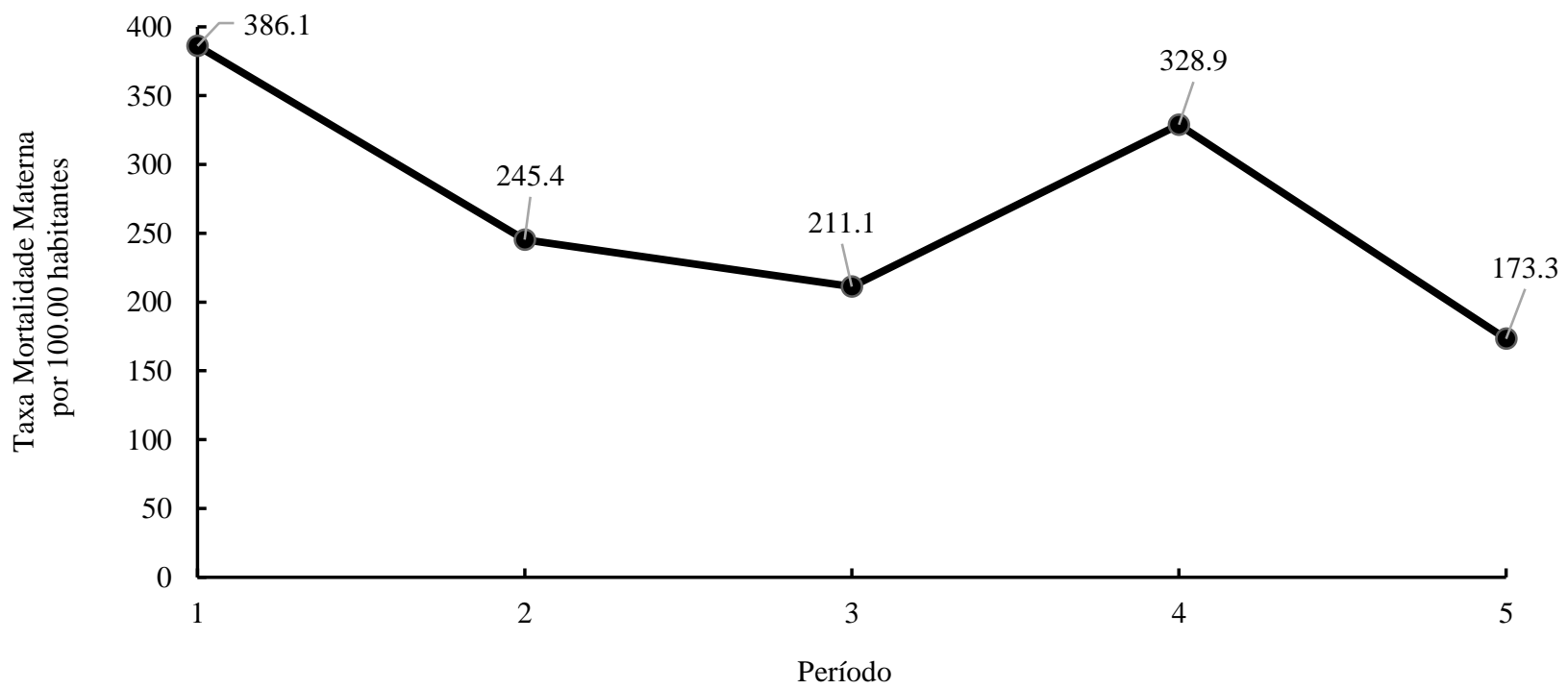

Fonte: Figueiredo LCA, et al., 2020; Dados Extraídos do Sistema de Informação de Mortalidade (SIM).

Assim, foi representada a distribuição espacial dos óbitos maternos conforme as quatro macrorregiões do Espírito Santo ao longo dos anos de 2014 a 2018 (Figura 1). Nesta análise, foi observado o total acumulado por cada macrorregião no período estudado e, portanto, foi identificado que o Sul apresenta a maior taxa acumulada de óbitos, referente à 388.3 óbitos/100.000 habitantes, acompanhada da macrorregião norte que registrou 380.2 óbitos/100.000 habitantes. Foi possível analisar que a macrorregião Metropolitana apresenta a menor taxa de mortalidade acumulada 269.9 óbitos/100.000 habitantes. A tonalidade cinza mais escura no mapa evidencia uma maior incidência de mortalidade materna na macrorregião, enquanto as tonalidades mais claras demonstram um número menor de óbitos maternos. 
Figura 1 - Incidência de Mortalidade Materna por 100.000 habitantes nas macrorregiões do estado do Espírito Santo.

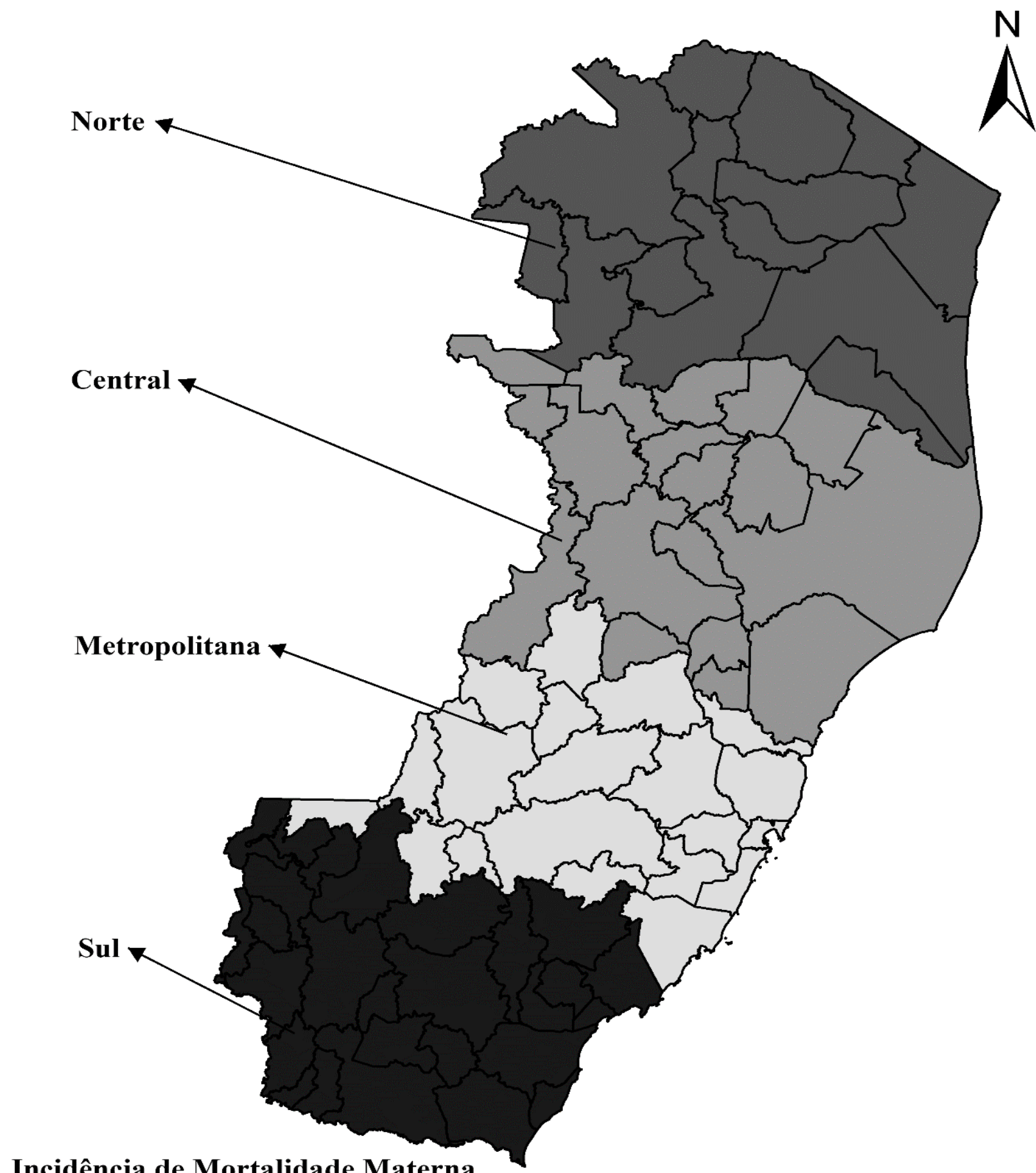

Incidência de Mortalidade Materna por 100.000 habitantes

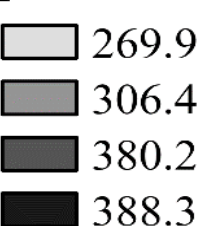

306.4

388.3

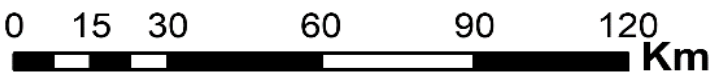

Legenda: As tonalidades de cinza representam a incidência de mortalidade materna, quanto maior a intensidade de cinza maior a incidência de óbitos maternos.

Fonte: Figueiredo LCA, et al., 2020; Dados Extraídos do Sistema de Informação de Mortalidade (SIM) e do Sistema de Informação Geográfica (SIG). 


\section{DISCUSSÃO}

Neste trabalho, foi identificado um perfil de gestante que parece estar associado à maior mortalidade materna no estado do Espírito Santo. Trata-se, predominantemente, das mulheres de pele parda, com 8 a 11 anos de escolaridade, solteiras e que possuem faixa etária de 30 a 39 anos. No que diz respeito a localização, a macrorregião Sul obteve os maiores números de mortes maternas enquanto a macrorregião Metropolitana totalizou a menor incidência de mortalidade materna.

Segundo os dados do IBGE de 2019, 50.5\% da população do Espírito Santo é parda (IBGE, 2019). No presente estudo, $47.09 \%$ das gestantes se declararam pardas. Costa LD, et al. (2016) também analisaram o perfil epidemiológico de 61 gestantes no Paraná e identificaram que $56,8 \%$ das puérperas eram pardas. Dessa mesma maneira, Esposti CDD, et al. (2015), em um estudo com 24 puérperas da região metropolitana da Grande Vitória - Espirito Santo, relataram que 14 mulheres declaram-se pardas ou pretas.

Em dissonância, trabalhos como o de Pimentel K, et al. (2012), na análise de 104 prontuários, identificaram que a cor branca da pele foi registrada para $43,3 \%$ das mulheres, seguida de parda, com $42,1 \%$. Esses dados sugerem que as gestantes pardas representam realmente a maioria racial dentro da população de grávidas. Além disso, é importante ressaltar que esses dados de cor e raça podem ser subjetivos, já que a classificação da cor da pele é realizada a partir da autodeclaração do indivíduo que responde à pergunta do prontuário (ANJOS JCS, et al., 2014).

$\mathrm{Na}$ análise da variável escolaridade foi possível identificar que a faixa de 8 a 11 anos de estudo, que representa a conclusão do Ensino Fundamental até o Ensino Médio, foi a predominante entre as gestantes. No Brasil, o grau de escolaridade médio é de 9,3 anos de estudo, conforme dados do Instituto de Geografia e Estatística (IBGE, 2018). Segundo o estudo de Silva MS e Rosa MRQP (2014), para 1510 prontuários de gestantes de alto risco, pode-se observar que 467 destas mulheres (30,9\%) haviam cursado o Ensino Médio. Já as pacientes com ensino fundamental completo e fundamental incompleto somam $48.4 \%$ (SILVA MS, ROSA MRQP, 2014), o que sugere que a baixa escolaridade possa incrementar o risco da gestação.

Outro estudo, realizado em Fortaleza - Ceará, também demonstrou uma semelhança nos resultados quanto à escolaridade: das 310 gestantes que frequentam o serviço de pré-natal nos Centros de Saúde da Família, em relação à escolaridade, predominou a faixa de 9 a 12 anos de estudo (146; 47,0\%) (PEIXOTO $\mathrm{CR}$, et al., 2012). Sendo assim, a escolaridade pode estar relacionada com o manejo da gravidez, seja ela precoce ou não. Além disso, Gomes RMT e César JA (2013), em um estudo transversal com questionário aplicado às gestantes no domicílio e na Unidade Básica de Saúde de Porto Alegre, Rio Grande do Sul, dentre as 238 gestantes entrevistadas, $38 \%$ possuía nove ou mais anos de estudo.

Apesar disso, é importante lembrar que a maioria das jovens que se encontram grávidas não frequentam a escola, sendo que o abandono escolar ocorre em sua maior parte antes da gestação, afastando-as das informações básicas sobre prevenção de gravidez que poderiam ser ofertadas em ambiente escolar (ANJOS JCS, et al., 2014). Dessa forma, é importante compreender que o nível de instrução da gestante pode influenciar no entendimento das informações fornecidas durante a consulta pré-natal, e isso reflete no cuidado com a família e com a gestação.

A respeito da faixa etária, verifica-se que o percentual de óbitos maternos é maior entre as mulheres de 30 a 39 anos; resultado que vem ao encontro da pesquisa de Riquinho DL e Correia SG (2006), que objetivou avaliar a morte materna na cidade de Porto Alegre (RS), e identificou que os óbitos concentram-se na faixa etária dos 30 aos 39 anos (46,2\%), e dos 20 aos 29 anos de idade (43,6\%). Nesse sentido, o estudo de Martins ACS e Silva LS (2018) também se assemelha a esses resultados: foram identificadas e analisadas as 85 mortes de gestantes residentes em Juiz de Fora, e a faixa etária compreendida com $80 \%$ dos óbitos registrados foi entre 20 e 40 anos. Dessa forma, é válido ressaltar que a faixa etária em que se registra mais óbitos maternos coincide com o período de maior atividade reprodutiva da mulher, isto é, entre 20 e 39 anos (ANDRADE ATL, et al., 2006).

A análise relacionada ao estado civil das gestantes no Espírito Santo mostrou que os óbitos prevalecem nas mães solteiras $(49.0 \%)$ em detrimento das gestantes casadas (27.3\%). Isto é, deve-se considerar que o 
fato de ser mãe solteira abre precedente para uma desvantagem psicológica e cria uma tendência de menor estabilidade econômica, uma vez que as mudanças causadas pela chegada do recém-nascido não se resumem apenas aos aspectos psico-biológicos, mas também a fatores socioeconômicos (ANJOS JCS, et al., 2014).

O estudo de Pinto LF, et al. (2005), realizado com 69 gestantes residentes em Teresópolis - Rio de Janeiro, indicou que a falta de um companheiro também é um fator dificultador para algumas dessas gestantes, sendo que, entre as adolescentes, $22.7 \%$ se encontram nessa situação. Dessa forma, é necessário observar uma pressão social sobre mulher ao que se diz respeito à maternidade, uma vez que quando a gravidez não é desejada e nem compartilhada há um conflito pessoal que se estende desde que a gravidez é anunciada até o período do parto (NASCIMENTO RJ, 2019). Assim, ao considerar este fato, nota-se que muitas mulheres sofreram e morreram por terem que assumir os desafios e responsabilidades da gestação que não eram só delas.

Na macrorregião Sul pode-se observar uma maior incidência de óbitos maternos, enquanto a macrorregião Metropolitana apresentou a menor taxa de mortalidade materna no período estudado. Pazó RG, et al. (2017) apresentaram um estudo cujos resultados reiteram esses achados: verificou-se que a maior taxa de Internações por Condições Sensíveis à Atenção Primária (ICSAP) ocorreu na macrorregião Sul e a menor na Metropolitana, fenômeno que se explica devido à maior oferta de leitos na macrorregião Sul. É pertinente comentar que, embora esta macrorregião tenha o PIB per capita elevado graças à arrecadação de royalties de petróleo, isso não significa que haja maior investimento em ações de promoção e prevenção de saúde próprias da Atenção Primária à Saúde (APS).

Desse modo, é importante salientar que no Espírito Santo ocorreram significativas mudanças socioeconômicas e estas precisam ser consideradas ao avaliar as condições de atenção à saúde da mulher. A modernização ocorrida na macrorregião Metropolitana causou impactos sociais, culturais, políticos e psicológicos que podem influenciar na adaptação das camadas sociais e dos indivíduos, bem como criar equilíbrios instáveis, decorridos da transformação do modo de vida tradicional (RODRIGUES MBF, CRUZ DS, 2011).

Os serviços de maternidade no Brasil enfrentam desafios crônicos como escassez de profissionais e de recursos (BITTENCOURT SDA, et al., 2016). Assim faz-se necessário a implementação de políticas públicas direcionada à atenção da saúde da mulher, com atendimento integral desde o pré-natal ao parto e puerpério. O atual o cenário de crise da saúde pública causado pela pandemia do coronavírus reiterou essas necessidades: Takemoto MLS, et al. (2020) mostraram que, dentre as 160 mortes maternas atribuídas ao Covid-19 no mundo, o Brasil foi responsável por 124. Isso significa que dos 188 territórios afetados pela pandemia do coronavírus, o Brasil registra mais mortes maternas que a soma de todos esses países.

Esse estudo apresenta como limitação a subnotificação de dados, pois alguns dados podem ser ignorados durante o preenchimento do formulário e, portanto, não há o registro completo de algumas variáveis estudadas no banco de dados do DATASUS. Apesar dessa limitação, o resultado é relevante ao se atentar para a saúde da mulher gestante segundo características sociodemográficas e econômicas. Não há financiadores para este trabalho.

\section{CONCLUSÃO}

Dessa maneira, a negligência do acompanhamento da gestante no Brasil não é uma questão inédita, mas sim uma adversidade presente na rede de saúde pública do país, que necessita de atenção e resolução. Investimentos em saúde pública com foco na ampliação e acessibilidade das gestantes ao pré-natal são primordiais para se reduzir o óbito nesse grupo, uma vez que o diagnóstico precoce permite identificar as causas evitáveis da mortalidade materna. De acordo com este estudo, os resultados mostram importantes informações sobre o estado do Espírito Santo e suas macrorregiões. Portanto, espera-se que esta pesquisa possa contribuir para o enfrentamento da problemática da mortalidade materna no estado. 


\section{REFERÊNCIAS}

1. ALVES KCG, ZANDONADE E. Tendências da mortalidade infantil no Estado do Espírito Santo, Brasil, 1979 a 2004. Rev. APS, 2009; 12(3): 302-310.

2. ANDRADE ATL, et al. Mortalidade materna: 75 anos de observações em uma Maternidade Escola. Rev Bras Ginecol Obstet., 2006; 28(7): 380-387.

3. ANJOS JCS, et al. Perfil epidemiológico das gestantes atendidas em um centro de referência em pré-natal de alto risco. Rev para med., 2014; 28(2): 23-32.

4. BITTENCOURT SDA, et al. Adequacy of public maternal care services in Brazil. Reprod Health, 2016; 13(S3): 120.

5. BRASIL. Estudo da mortalidade de mulheres de 10 a 49 anos, com ênfase na mortalidade materna: relatório final. Brasília: Ministério da saúde; 2006.

6. BRASIL. Portaria oㅜ 1.459, de 24 de junho de 2011: Brasília: Ministério da saúde; 2011.

7. COSTA LD, et al. Perfil Epidemiológico De Gestantes De Alto Risco. Cogitare Enfermagem 2016; 21(2):01-08.

8. DIAS JMG, et al. Mortalidade materna. Rev Assoc Med Minas Gerais, 2014; 25(2): 173-179.

9. ESPOSTI CDD, et al. Representações sociais sobre o acesso e o cuidado pré-natal no Sistema Único de Saúde da Região Metropolitana da Grande Vitória, Espírito Santo. Saúde e Sociedade, 2015; 24(3): 765-779.

10. GOIS EC, et al. Mortalidade materna na Bahia no período de 2012 a 2016. Revista Eletrônica Acervo Saúde, 2019; (18): e335.

11. GOMES RMT, CÉSAR JA. Perfil epidemiológico de gestantes e qualidade do pré-natal em unidade básica de saúde em Porto Alegre, Rio Grande do Sul, Brasil. Revista Brasileira de Medicina de Família e Comunidade, 2013; 8(27): 80-89.

12. INSTITUTO BRASILEIRO DE GEOGRAFIA E ESTATÍSTICA (IBGE). $2019 . \quad$ Disponível em: https://cidades.ibge.gov.br/brasil/es/pesquisa/10070/64506?indicador=62929\&ano=2019. Acesso em: 11 ago. 2020.

13. INSTITUTO BRASILEIRO DE GEOGRAFIA E ESTATÍSTICA (IBGE). Pesquisa Nacional por Amostra de Domicílios $\begin{array}{llll}\text { Contínua. } & \text { Contínuar } & 2018 . & \text { Disponível }\end{array}$ https://biblioteca.ibge.gov.br/visualizacao/livros/liv101657_informativo.pdf. Acesso em: 07 set. 2020.

14. LAURENTI R, et al. A mortalidade materna nas capitais brasileiras: algumas características e estimativa de um fator de ajuste. Rev Bras Epidemiol., 2004; 7(4): 449-460.

15. LAURENTI R, et al. Mortes maternas e mortes por causas maternas. Epidemiol Serv Saúde, 2008; 17(4):283-292.

16. MARTINS ACS, SILVA LS. Epidemiological profile of maternal mortality. Revista Brasileira de Enfermagem, 2018; 71677-683.

17. MATIAS JP, et al. Comparação entre dois métodos para investigação da mortalidade materna em município do Sudeste brasileiro. Rev Bras Ginecol Obstet., 2009; 31(11): 559-565.

18. MONTE AS, et al. Near miss materno: fatores influenciadores e direcionamentos para redução da morbidade e mortalidade materna. Rev. RENE, 2018; 19: e3182-e3182.

19. NASCIMENTO RJ. Solteiras sim, mãe também: os desafios diários das mulheres "mães solteiras" de Ponta do Mel/RN. Monografia - Universidade Federal Rural do Semi-Árido. Mossoró; 2019.

20. NOGUEIRA LDP, OLIVEIRA GS. Assistência pré-natal qualificada: As atribuições do enfermeiro. Rev Enferm Atenção Saúde, 2017; 6(1):107-119.

21. PAZÓ RG, et al. Panorama das internações por condições sensíveis à atenção primária no Espírito Santo, Brasil, 2000 a 2014. Revista Brasileira de Medicina de Família e Comunidade, 2017; 12(39): 1-12.

22. PEIXOTO CR, et al. Perfil das gestantes atendidas no serviço de pré-natal das unidades básicas de saúde de Fortaleza-CE. REME, 2012; 16(2): 171-177.

23. PIMENTEL K, et al. Perfil clínico-social das gestantes atendidas numa unidade docente-assistencial baseada no modelo de saúde da família. Revista Baiana de Saúde Pública, 2012; 35(2): 239-239.

24. PINTO LF, et al. Perfil social das gestantes em unidades de saúde da família do município de Teresópolis. Cien Saude Colet., 2005; 10(1): 205-213.

25. RIQUINHO DL, CORREIA SG. Mortalidade materna: perfil sócio-demográfico e causal. Revista Brasileira de Enfermagem, 2006; 59(3): 303-307.

26. RODRIGUES MBF, CRUZ DS. Políticas públicas e gestão urbana: o caso da região metropolitana da Grande Vitória no estado do Espírito Santo. Dim, 2011; (27): 23-39.

27. SANTOS DR, et al. Maternal mortality in the indigenous and non-indigenous population in Pará: contribution to the surveillance of deaths. Escola Anna Nery, 2017; 21(4): e20170161.

28. SILVA MS, ROSA MRQP. Perfil de gestantes de alto risco atendidas em um centro obstétrico de Santa Catarina. Revista Interdisciplinar, 2014; 7(2): 95-102.

29. SOUZA JP. Maternal mortality and the new objectives of sustainable development (2016-2030). Rev Bras Ginecol Obstet., 2015; 37(12): 549-551.

30. TAKEMOTO MLS, et al. The tragedy of COVID-19 in Brazil: 124 maternal deaths and counting. Obstet Gynecol Int J., 2020; 2377-4304.

31. UNITED NATIONS DEVELOPMENT PROGRAMME (UNDP). The Millennium Development Goals Report 2014. New York: United Nations; 2014.

32. UNITED NATIONS POPULATION FUND (UNFPA). The Social Determinants of Maternal Death and Disability. UNFPA; 2012.

33. WORLD HEALTH ORGANIZATION (WHO), UNICEF, United Nations Fund for Population Activities, World Bank. Trends in maternal mortality: 1990 to 2010. Geneva, Switzerland: World Health Organization; 2012. 